\title{
In Vitro Analysis of Licorice (Glycyrrhiza glabra) Root Extract Activity on Streptococcus mutans in Comparison to Chlorhexidine and Fluoride Mouthwash
}

\author{
Ekta A Malvania ${ }^{1}$, Ashish S Sharma ${ }^{2}$, Sona A Sheth ${ }^{3}$, Sanjay Rathod ${ }^{4}$, Nishant R Chovatia ${ }^{5}$, Mansoor S Kachwala ${ }^{6}$
}

\begin{abstract}
Aim: The present study was done to determine the activity of licorice root extract on Streptococcus mutans (S. mutans) in comparison to chlorhexidine and fluoride mouthwash.

Materials and methods: In the current study, the different concentrations of aqueous and ethanolic licorice root extract were subjected to microbiological assay and zone of inhibition was determined against S. mutans by agar ditch method. Minimum inhibitory concentration (MIC) of aqueous and ethanolic solution was obtained by using broth dilution method and agar dilution method. Chlorhexidine and fluoride mouthwash were kept as a positive control in the present study. One-way ANOVA along with Tukey post hoc test were used at 5\% level of significance to analyze data.

Results: Mean zone of inhibition of chlorhexidine mouthwash, fluoride mouthwash, aqueous and ethanolic licorice root extracts against $S$. mutans at 24 hours were $23 \mathrm{~mm}, 14.2 \mathrm{~mm}, 15.8 \mathrm{~mm}$ and $22.4 \mathrm{~mm}$, respectively. Minimum inhibitory concentration of aqueous and ethanolic licorice root extract on S. mutans was $20 \mathrm{mg} / \mathrm{mL}$ and $12.5 \mathrm{mg} / \mathrm{mL}$, respectively by both broth dilution method and agar dilution method.

Conclusion: The antibacterial effect produced by ethanolic licorice root extract on S. mutans was comparable to chlorhexidine mouthwash while significantly higher in comparison with aqueous form and fluoride mouthwash.

Clinical significance: The interest in the plants with antibacterial and anti-inflammatory activity has increased now days to treat various dental diseases as consequences of current problems associated with the conventional agents. Licorice root is easily available, economically feasible and culturally acceptable and may possess minimal side effects as compared to conventional means of chemicotherapeutic agents used for reduction of $S$. mutans in oral cavity and hence can be recommended for prevention of dental caries.

Keywords: Chlorhexidine, Dental caries, Fluoride mouthwash, In vitro study, Licorice root.

The Journal of Contemporary Dental Practice (2019): 10.5005/jp-journals-10024-2724
\end{abstract}

\section{INTRODUCTION}

Dental caries is a common problem faced by mankind which poses a serious threat to dental healthcare. ${ }^{1}$ The ongoing research for prevention of dental caries is mainly intent upon the techniques to eliminate cariogenic flora from the oral cavity. Streptococcus mutans play a major role in initiation and progression of dental caries. However, it is difficult to abolish S. mutans from tooth surface by mechanical measures alone. Mechanical and chemical plaque control methods when used in adjunct can achieve maximum microbial count reduction. The action of chlorhexidine, fluoride mouthwash and antibiotics is either by minimizing the plaque formation or by reducing the microflora. Nevertheless, the adverse side effects of these agents like tooth discoloration, irritating effect and development of bacterial resistance will be consequently leading to degradation of the efficacy of conventional medicines, thus triggering the exploration of other agents. ${ }^{2}$

In India, Ayurveda is considered as an ancient medicinal form, existing since $2000 \mathrm{BC}$. Many drugs used in allopathic medicines as well as alternative medicines have their origin in medicinal plants as they are well recognized source of potential bioactive compounds. ${ }^{3}$ Use of plants can be justified because of less cost, easy availability, easy absorption with no or minimal adverse effect. ${ }^{4}$ There have been numerous reports of use of traditional plants and their products for treatment of oral diseases. Many plants and chewing sticks were reported to inhibit the growth of many microorganisms mainly S. mutans, thus can help in prevention of dental caries. ${ }^{5}$ \begin{tabular}{l}
\hline \hline${ }^{1-3}$ Department of Public Health Dentistry, AMC Dental College and \\
Hospital, Ahmedabad, Gujarat, India \\
${ }^{4}$ Department of Microbiology, AMC MET Medical College and LG \\
Hospital, Ahmedabad, Gujarat, India \\
${ }^{5,6}$ Department of Public Health Dentistry, College of Dental Science
\end{tabular} 5,6 Department of Public Health Dentistry,
and Research Centre, Ahmedabad, India

Corresponding Author: Ekta A Malvania, Department of Public Health Dentistry, AMC Dental College and Hospital, Ahmedabad, Gujarat, India, Phone: +91 9925367532, e-mail: drektadamani@gmail.com

How to cite this article: Malvania EA, Sharma AS, Sheth SA, et al. In Vitro Analysis of Licorice (Glycyrrhiza glabra) Root Extract Activity on Streptococcus mutans in Comparison to Chlorhexidine and Fluoride Mouthwash. J Contemp Dent Pract 2019;20(12):1389-1394.

Source of support: Nil

Conflict of interest: None

Glycyrrhiza glabra (licorice) which is also known as Yashti Madhu in Hindi and Jethi Madhu in Gujarati is having its own medicinal value. The Egyptians, Greeks and Romans have utilized this medicine to treat different types of illness. ${ }^{6}$ Licorice contains more than 20 titerpenoids and nearly 300 flavonoids. Among them glycyrrhizin (GL), 18- $\beta$-glycyrretinic (GA), liquitigenin (LTG), licochalcone $A(L C A)$, licochalcone $E(L C E)$, licorisoflavan $A$ and glabridin (GLD) are main active components which possess antimicrobial and antiviral activities. ${ }^{7,8}$ The cork layer of rhizome

o The Author(s). 2019 Open Access This article is distributed under the terms of the Creative Commons Attribution 4.0 International License (https://creativecommons. org/licenses/by-nc/4.0/), which permits unrestricted use, distribution, and non-commercial reproduction in any medium, provided you give appropriate credit to the original author(s) and the source, provide a link to the Creative Commons license, and indicate if changes were made. The Creative Commons Public Domain Dedication waiver (http://creativecommons.org/publicdomain/zero/1.0/) applies to the data made available in this article, unless otherwise stated. 
of licorice shows a powerful antibacterial activity against gramnegative and gram-positive bacteria. ${ }^{9}$ According to study reported by Prusti et al. ${ }^{10}$ minimal two compounds derived from the licorice plant root were considered as potent inhibitors of S. mutans. Hence, the present study was carried out to analyze the activity of aqueous and ethanolic licorice root extract on S. mutans. Chlorhexidine and fluoride mouthwash were kept as a positive control in the study.

\section{Materials and Methods}

\section{Plant Material}

\section{Procurement of Licorice Root}

Roots of licorice were purchased from local ayurvedic store of Ahmedabad, Gujarat, India. The species were identified and authenticated by the Department of Botany, Gujarat University, Ahmedabad (Fig. 1).

\section{Place of the Study and Related Approvals}

The present study was carried out in the Department of Microbiology; AMC MET Medical College and LG Hospital, Ahmedabad. Prior approval of Dean of AMC Dental College, AMC MET Medical College and LG Hospital, Head of the Department of Department of Microbiology, AMC MET Medical College and LG Hospital and Institutional Review Board of AMC Dental College were taken to conduct the study.

Preparation of Aqueous and Ethanolic Extract of Licorice Root Roots of plant were sun dried for 2 days and powdered using a mechanical grinder. Aqueous solution of licorice root was prepared by mixing $30 \mathrm{~g}$ of dry powder in $150 \mathrm{~mL}$ of distilled water in a flask with gentle shaking. Ethanolic solution of licorice root was prepared by mixing $30 \mathrm{~g}$ of dry powder in $150 \mathrm{~mL}$ of $70 \%$ (w/v) ethyl alcohol for 7 days in a flask with gentle shaking. After that, the filtration of aqueous and ethanolic extract was done firstly through a muslin cloth for coarse residue and finally through Whatman no. 1 filter paper. Both the prepared solutions were then kept in an airtight amber colored container and stored at $4^{\circ} \mathrm{C}$ for further use (Fig. 2).

\section{Microbial Analysis}

The microbial strain used in the study was S. mutans American type culture collection (ATCC) 25175. The strain was collected from ATCC, USA (Fig. 3).

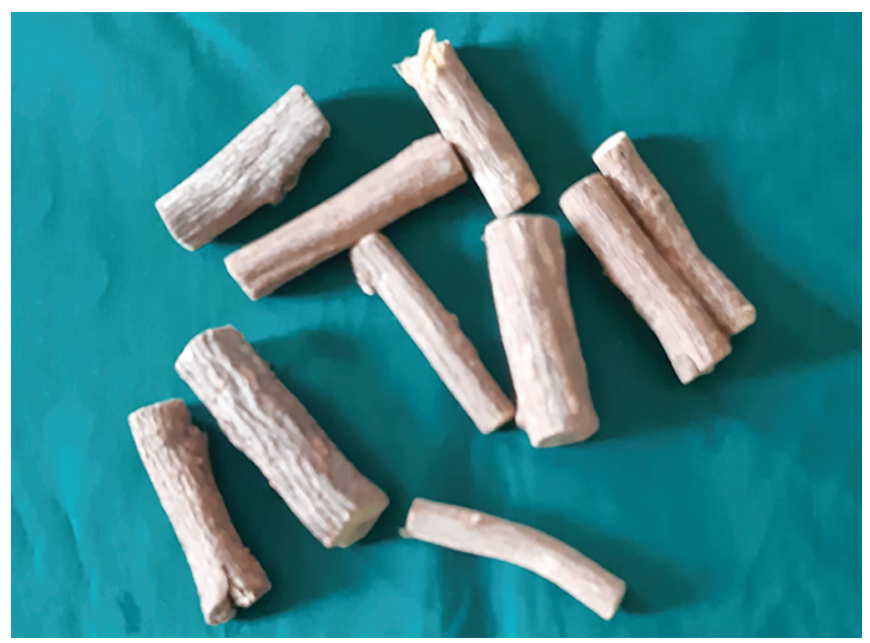

Fig. 1: Licorice root specimen used in the present study

\section{Revival of Organisms}

Brain heart infusion (BHI) broth was used to reconstitute freeze dried form of bacterial strains. Reconstituted bacterial strains were revived by plating on blood agar medium and incubated at $37^{\circ} \mathrm{C}$ under capnophilic condition in candle jar for 48 hours. After confirming the identities of organisms, colonies were picked up from appropriately incubated agar cultures to sterile BHI broth to make suspension of test organisms and once again incubation was done overnight. The growth concentration was adjusted to match McFarland 0.5 turbidity standard (approximately $1.5 \times 10^{8} \mathrm{cfu} / \mathrm{mL}$ ).

\section{Agar Ditch Plate Method for Evaluating the Antibacterial Properties}

By using swab technique, approximately $100 \mu \mathrm{L}$ of S. mutans strain were inoculated on Petri dish containing $15 \mathrm{~mL}$ of BHI agar. With the help of a sterilized standard device, wells of $8 \mathrm{~mm}$ diameter were cut into solidified agar media and in the respective well, $100 \mu \mathrm{L}$ of aqueous licorice root extract, ethanolic root extract, chlorhexidine mouth wash and fluoride mouthwash were poured. The plates were then subjected to incubation at $37^{\circ} \mathrm{C}$ for 24 hours. To enhance the efficacy of the results, the experiment was conducted three times under stern sterile condition. The antibacterial activity of each extract was shown through mean of diameter of zone of inhibition (in $\mathrm{mm}$ ) produced by each extract at the end of incubation period (Fig. 4).

\section{Minimum Inhibitory Concentration determination by Broth Dilution Method}

A total of 8 test tubes having total $2 \mathrm{~mL}$ solution of $\mathrm{BHI}$ and licorice root extract was prepared which shows concentration of active licorice root extract ranging from 50, 35, 30, 20, 12.5, 6, 4, $2.5,1.25 \mathrm{mg} / \mathrm{mL}$. To each 8 of such prepared tubes with different concentration, $2 \mathrm{~mL}$ of already constituted suspension of $S$. mutans was supplemented in such a manner that the ultimate volume of tube came out to be $4 \mathrm{~mL}$. One test tube containing only broth and S. mutans strain was kept as positive control and one test tube containing only broth and licorice solution was kept as negative control. All test tubes were incubated at $37^{\circ} \mathrm{C}$ for 24 hours.

To determine the MIC of extract more accurately and for confirmation of results, agar dilution method was used.
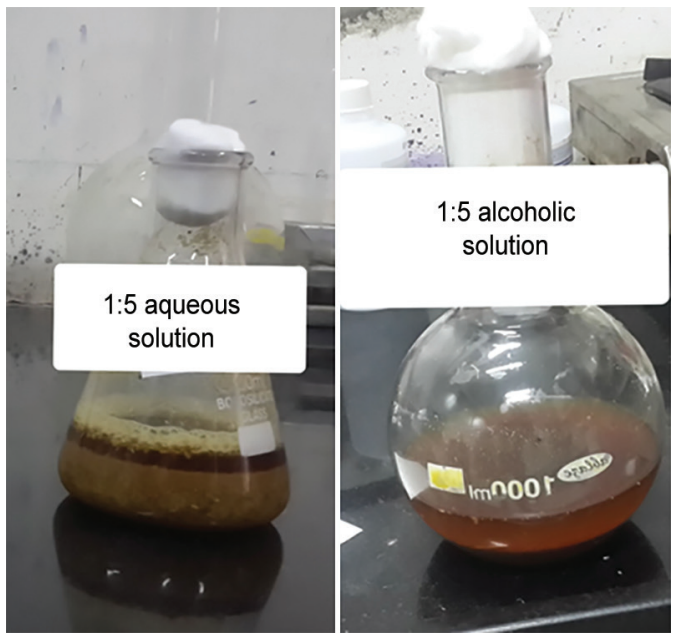

Fig. 2: Aqueous and ethanolic licorice root extract solution presented in the current study 


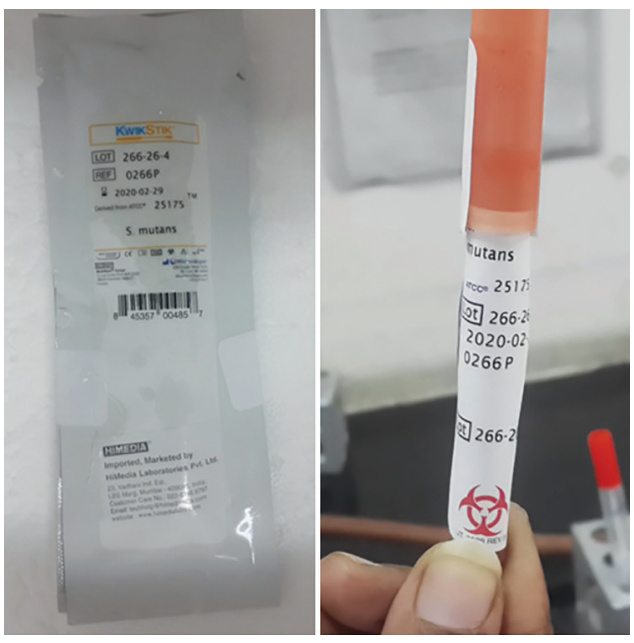

Fig. 3: Streptococcus mutans strain ATCC 25175

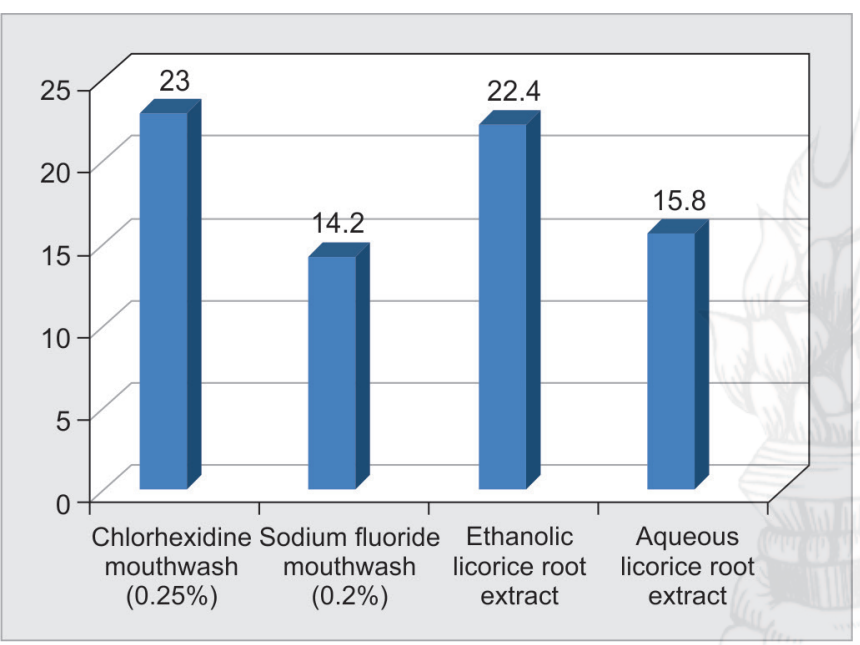

Fig. 5: Mean zone of inhibition of different solutions on Streptococcus mutans at 24 hours by agar ditch method

\section{Minimum Inhibitory Concentration determination by Agar Dilution Method}

Agar dilution assay was used to assess the susceptibility of S. mutans to licorice extract at different concentration, as recommended by the Clinical Laboratory Standards Institute (CLSI). ${ }^{11}$ Dilutions of licorice root extract were formulated in Mueller-Hinton agar ( $\mathrm{MH}$ agar) plates according to standard procedures. After solidification, the plates were incubated at $37^{\circ} \mathrm{C}$ for 2 hours in order to dry the agar surface.

The assay plates were estimated to have $50,35,30,20,12.5,10$, $6,4,2.5,1.25 \mathrm{mg} / \mathrm{mL}$ active licorice extract. Inocula were applied to agar surface in $1 \mathrm{sq} . \mathrm{cm}$. Plates without added extract were inoculated as positive control. All plates were incubated at $37^{\circ} \mathrm{C}$ for 24 hours. The MIC was considered as the lowest concentration of extract which shows no growth of organism, as compared to positive control.

All the agents were given code during procedure. Coding and decoding of agents were done by the laboratory person who was unaware about the objectives of the study. Zone of inhibition were measured by two examiners. Inter examiner variability was calculated by re-examining $5 \%$ of samples, which gives kappa value of 0.86 (almost perfect agreement).

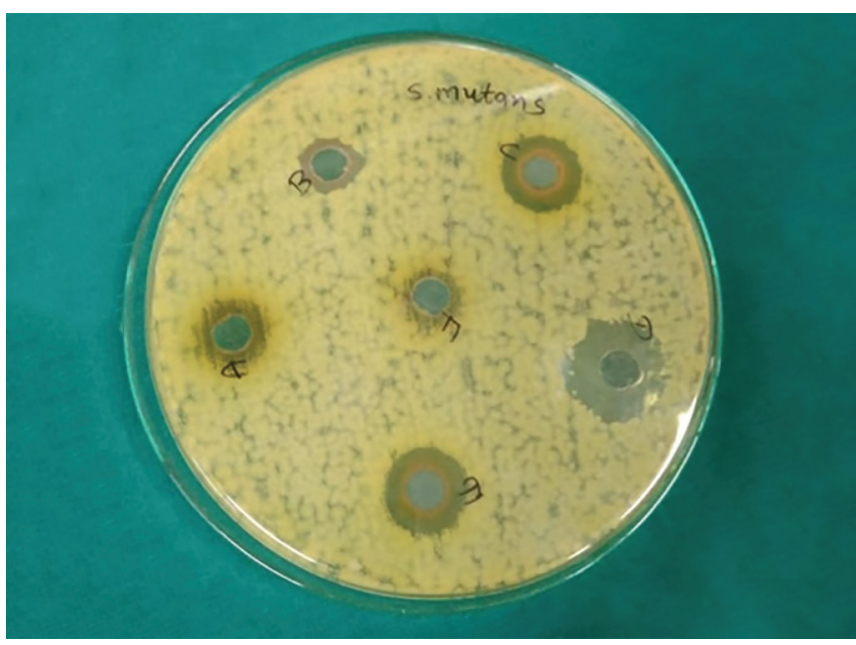

Fig. 4: Antimicrobial activity of different solutions against Streptococcus mutans at 24 hours by using agar ditch method: (A) Chlorhexidine mouthwash (0.2\%); (B) Sodium fluoride mouthwash (0.2\%); (C) Aqueous licorice root extract [prepared by dissolving $30 \mathrm{~g}$ of dry powder in 150 $\mathrm{mL}$ of distilled water (1:5)]; (D) Ethanolic licorice root extract [prepared by dissolving $30 \mathrm{~g}$ of dry powder in $150 \mathrm{~mL}$ of $70 \%$ (w/v) ethyl alcohol (1:5)]; (E and F) [Solutions which are not presented in the current study where $(E)$ is aqueous licorice root extract prepared by dissolving $30 \mathrm{~g}$ of dry powder in $300 \mathrm{~mL}$ of distilled water (1:10); (F) Ethanolic licorice root extract prepared by dissolving $30 \mathrm{~g}$ of dry powder in $300 \mathrm{~mL}$ of $70 \%(w / v)$ ethyl alcohol (1:10)]

\section{Statistical Analysis}

The data were analyzed by using SPSS version 17. Multiple group comparison was done by applying one-way ANOVA test, subsequence by Tukey post hoc for groupwise comparison. Level of significance was kept at $5 \%$.

\section{Results}

At the end of 24 hours, all the solutions showed anti-microbial activity against $S$. mutans.

Figure 5 shows mean zone of inhibition of different solutions on S. mutans at 24 hours by agar ditch method. Amongst all the solutions, inhibitory effect produced by chlorhexidine mouthwash against $S$. mutans was almost similar to ethanolic licorice root extract, while much higher when compared to aqueous licorice root extract and sodium fluoride mouthwash.

Table 1 reveals antibacterial activity of different solutions on S. mutans at 24 hours by agar ditch method. Multiple groupwise comparison showed statistically significant difference amongst different groups. To assess groupwise significant difference, Tukey post hoc test was performed which showed that inhibitory effect produced by chlorhexidine mouthwash and ethanolic licorice root extract against $S$. mutans were statistically higher than aqueous licorice root extract and sodium fluoride mouthwash. No statistically significant difference observed between chlorhexidine mouthwash and ethanolic licorice root extract and between aqueous licorice root extract and sodium fluoride mouthwash.

Table 2 shows MIC of aqueous licorice root extract on S. mutans by broth dilution method and agar dilution method. The results of broth dilution method and agar dilution method are comparable. By both methods, $S$. mutans showed sensitivity to aqueous licorice root extract to $50 \mathrm{mg} / \mathrm{mL}, 35 \mathrm{mg} / \mathrm{mL}, 30 \mathrm{mg} / \mathrm{mL}$ and $20 \mathrm{mg} / \mathrm{mL}$ 
Table 1: Antibacterial activity of different solutions on Streptococcus mutans at 24 hours by agar ditch method

\begin{tabular}{lccl}
\hline Different solutions & $\begin{array}{c}\text { Mean zone of inhibition in } \mathrm{mm} \\
(\text { mean } \pm \text { SD) }\end{array}$ & $\begin{array}{l}\text { p value for one way } \\
\text { ANOVA test }\end{array}$ & $\begin{array}{l}\text { Tukey post hoc (group wise } \\
\text { significant difference) }\end{array}$ \\
\hline Chlorhexidine mouthwash $(0.2 \%)$ & $23 \pm 1.5$ & $0.0001^{*}$ & $1>2$ \\
Sodium fluoride mouthwash $(0.2 \%)$ & $14.2 \pm 0.6$ & & $1>4$ \\
Ethanolic licorice root extract & $22.4 \pm 0.7$ & $3>2$ \\
Aqueous licorice root extract & $15.8 \pm 0.2$ & $3>4$ \\
\hline
\end{tabular}

${ }^{*} p<0.01$, statistically highly significant; SD, standard deviation; 1 -chlorhexidine mouthwash (0.2\%); 2-sodium fluoride mouthwash (0.2\%), 3-ethanolic licorice root extract; 4-aqueous licorice root extract

${ }^{*}$ Results for Tukey post hoc test for group wise comparison

$p<0.05$ for group wise comparison between chlorhexidine mouthwash and sodium fluoride mouth wash and between chlorhexidine mouthwash and aqueous licorice root extract

$p<0.05$ for group wise comparison between ethanolic licorice root extract and sodium fluoride mouthwash (0.2\%) and between ethanolic licorice root extract and aqueous licorice root extract

Table 2: Minimum inhibitory concentration of aqueous licorice root extract on Streptococcus mutans by broth dilution method and agar dilution method

\begin{tabular}{|c|c|c|c|c|c|c|c|c|c|c|c|}
\hline \multirow[b]{2}{*}{ Microorganism } & \multirow[b]{2}{*}{ Methods } & \multicolumn{10}{|c|}{ Concentration of aqueous licorice root extract in $\mathrm{mg} / \mathrm{mL}$} \\
\hline & & 50 & 35 & 30 & 20 & 12.5 & 10 & 6 & 4 & 2.5 & 1.25 \\
\hline \multirow[t]{2}{*}{ S. mutans } & Broth dilution & $\mathrm{S}$ & $\mathrm{S}$ & $\mathrm{S}$ & $\mathrm{S}$ & $\mathrm{R}$ & $\mathrm{R}$ & $\mathrm{R}$ & $\mathrm{R}$ & $\mathrm{R}$ & $\mathrm{R}$ \\
\hline & Agar dilution & $\mathrm{s}$ & $\mathrm{s}$ & $\mathrm{s}$ & $\mathrm{s}$ & $\mathrm{R}$ & $\mathrm{R}$ & $\mathrm{R}$ & $\mathrm{R}$ & $\mathrm{R}$ & $\mathrm{R}$ \\
\hline
\end{tabular}

$S$, sensitive to solution; $R$, resistant to solution

Table 3: Minimum inhibitory concentration of ethanolic licorice root extract on Streptococcus mutans by broth dilution method and agar dilution method

\begin{tabular}{|c|c|c|c|c|c|c|c|c|c|c|c|}
\hline \multirow[b]{2}{*}{ Microorganism } & \multirow[b]{2}{*}{ Methods } & \multicolumn{10}{|c|}{ Concentration of ethanolic licorice root extract in $\mathrm{mg} / \mathrm{mL}$} \\
\hline & & 50 & 35 & 30 & 20 & 12.5 & 10 & 6 & 4 & 2.5 & 1.25 \\
\hline \multirow[t]{2}{*}{ S. mutans } & Broth dilution & $\mathrm{S}$ & $\mathrm{S}$ & $\mathrm{S}$ & $S$ & $\mathrm{~S}$ & $\mathrm{R}$ & $\mathrm{R}$ & $\mathrm{R}$ & $\mathrm{R}$ & $\mathrm{R}$ \\
\hline & Agar dilution & $\mathrm{S}$ & $\mathrm{S}$ & $\mathrm{S}$ & $\mathrm{S}$ & $\mathrm{S}$ & $\mathrm{R}$ & $\mathrm{R}$ & $\mathrm{R}$ & $\mathrm{R}$ & $\mathrm{R}$ \\
\hline
\end{tabular}

$S$, sensitive to solution; $R$, resistant to solution

and started showing resistance from $12.5 \mathrm{mg} / \mathrm{mL}$ to $1.25 \mathrm{mg} / \mathrm{mL}$. Hence, MIC of aqueous licorice root extract was kept at $20 \mathrm{mg} / \mathrm{mL}$.

Table 3 reveals MIC of ethanolic licorice root extract on S. mutans by broth dilution method and agar dilution method. The results of both methods are similar. In both methods, S. mutans demonstrated sensitivity to ethanolic licorice root extract to the concentration of $50 \mathrm{mg} / \mathrm{mL}, 35 \mathrm{mg} / \mathrm{mL}, 30 \mathrm{mg} / \mathrm{mL}, 20 \mathrm{mg} / \mathrm{mL}$ and $12.5 \mathrm{mg} / \mathrm{mL}$ and started demonstrating resistance from $10 \mathrm{mg} / \mathrm{mL}$ to $1.25 \mathrm{mg} / \mathrm{mL}$. Hence, MIC of ethanolic licorice root extract was kept at $12.5 \mathrm{mg} / \mathrm{mL}$.

\section{Discussion}

Despite the advances in field of dentistry, dental caries continue to be a crucial public health problem and imposing a significant burden to healthcare services especially in developing countries. ${ }^{12}$ Caries initiation and progression occurs due to dental plaque and bacterial adherence to the susceptible tooth surface which produces acids from fermentation of dietary carbohydrate, ultimately leading to cavitation in the tooth. ${ }^{3}$

Prevention is better than cure. Dental caries prevention in an individual can be targeted either by enhancing the host capacity against response to any kind of insult or alleviating cariogenicity of bacteria and/or promoting less caries inducing food habits. ${ }^{3}$

In India, the use of different parts of several medicinal plants to cure specific ailments has been in vogue from ancient times. Licorice is a perennial herb or under shrub. It is used as traditional medicine for household remedy against various human ailments from antiquity. ${ }^{13}$ Licorice root is rich in many compounds, the pivotal being a glycoside, glycyrrhizin which possess a similar structure and activity as adrenal steroids. It possesses good antibacterial, anti-histaminic, anti-fungal, anti-oxidant and anti-inflammatory activity. ${ }^{14,15}$ In India, it is commonly used to treat respiratory problems, i.e., for allaying cough and to treat sore throat and as a tonic, diuretic, demulcent and laxative. ${ }^{3}$

Despite of its wide use in medical field, less literature is published on uses of licorice in dentistry. Hence, the present study was carried out to analyze the activity of aqueous and ethanolic licorice root extract on S. mutans.

Chlorhexidine and sodium fluoride mouthwash had been used as positive control in the present study which is most commonly used as an antimicrobial agent against S. mutans. Chlorhexidine gluconate, which is charged positively, has high affinity for negative ions found in cell membranes of microorganism. It instigates condensation of cytoplasmic protein and nucleic acid. It abolishes the function of phosphoenolpyruvate-phosphotransferase sugar transit system and hence significantly halts the acid production in oral streptococci. ${ }^{16}$ The effects of fluoride on streptococcal cells are partly ascribed to the inhibition of enolase enzyme and thus preventing conversion of glucose to lactic acid, thereby retards acid production in oral streptococci. ${ }^{17,18}$ At higher concentration, it might act as bactericidal. ${ }^{18}$ Various studies have shown sodium fluoride mouth rinse $(0.2 \%)$ to be effective in reducing S. mutans counts. ${ }^{19-22}$ 
A limited comparison of the present study to other studies can be done as from the available literature it has been found that only few in vitro studies have assessed the effects of licorice root on S. mutans.

In the present study, ethanolic licorice root extract showed anti-microbial activity comparable to that of $0.2 \%$ chlorhexidine mouthwash. Results are in agreement with the studies conducted by Lingaraj et al., ${ }^{3}$ Sedighinia et al., ${ }^{7}$ Geetha et al. ${ }^{14}$ and Ahn et al. ${ }^{23}$ It was unexpected that ethanolic licorice root extract would give significantly higher inhibitory effect than sodium fluoride mouthwash. Although fluoride is established as an effective anti caries agent, this is a first study of its kind which has compared the anti-bacterial activity of licorice root extract on S. mutans in comparison with sodium fluoride mouthwash.

The antibacterial activity of licorice might be attributed to one of its main ingredients, glycyrrhizin, which dose-dependently inhibits the glucosyltransferase activity of mutans streptococci, which is involved in the formation of insoluble glucans required in biofilm formation. ${ }^{24} \mathrm{Hu}$ et al. ${ }^{25}$ discovered a novel compound, glycyrrhizol A, from the extract of liquorice roots, which exerted strong antimicrobial action against cariogenic bacteria.

Amongst aqueous and ethanolic licorice root extract, ethanolic extract exhibited significantly higher inhibitory rates compared to aqueous form and hence showing less MIC value than aqueous form. Results are in comparison with the studies conducted by Lingaraj et al., ${ }^{3}$ Jain et al. ${ }^{26}$ This might be because of more effective dissolving capacity in alcohol, ${ }^{3}$ greater bio availability (hence intensifying bio activity) ${ }^{3}$ or polar nature of solvent (ethanol), which resulted in leaching of more active ingredients during extraction. ${ }^{26}$

\section{Conclusion and Clinical Significance}

The present study offers a scientific basis for the use of licorice root extract against S. mutans. The well-known side effects of chlorhexidine, i.e., staining of teeth and restoration and altered taste sensation may limit the long-term use of chlorhexidine. In comparison, licorice root is easily available, economically feasible and culturally acceptable and may possess minimal side effects and hence can be recommended for further use. Since, the present study is in vitro; the duration of the contact of the extract with microorganisms in the oral cavity in vivo is not established. Therefore, more scientific work needs to be carried out to prove the efficacy and for recommendation of ideal clinical protocols using this material especially in this era when holistic integrated medicine approach is the need of the hour. If further studies show promise, it can be used as mouthwash or as viscous suspension or gel as an anti caries agent. For infants, liquorice can be incorporated in infant swipes and pacifiers, which would serve as non cariogenic sweet tasting alternative to commonly used honey dipped pacifiers.

\section{ACKnOWLedgments}

The authors would like to thank Dean, AMC Dental College, Dean, AMC MET Medical College, Head of Department of Microbiology, AMC MET Medical College to permit us to conduct the study and all technician staffs of Department of Microbiology, AMC MET Medical College who helped us in the study.

\section{References}

1. Kavya R. Glycyrrhiza glabra - an ayurvedic medicine in dentistry. Res J Pharm Technol 2018;7(7):821-822.
2. Eley BM. Antibacterial agents in the control of supragingival plaque-A review. Br Dent J 1999;186(6):286-296. DOI: 10.1038/sj.bdj. 4800090.

3. Lingaraj S, Battur H, Shamarao S, et al. Effects of aqueous and alcoholic licorice root extract against $\mathrm{S}$. mutans and L. acidophilus in comparison to chlorhexidine: an in vitro study. J Int Oral Health 2014;6(4):29-34.

4. Hiregoudar M, Subramaniam R, Mittal S, et al. Antibacterial activity of some spice extract on Streptococcus mutans: an in vitro study. J Indian Assoc Public Health Dent 2011;17(1):336-340.

5. Kumar D, Sidhu P. The antimicrobial activity of Azardirachta indica, Glycyrrhiza glabra, cinnamum zaylaicum, syzygium aromaticum, acacia nilotica on Streptococcus mutans and enterococcus feacalis: an in vitro study. Int Endod J 2012;7:18-25.

6. Isbrucker RA, Burdock GA. Risk and safety assessment on the consumption of licorice root, it's extract and powder as a food ingredient, with emphasis on the pharmacology and toxicity of licorice. Regul Toxicol Pharmacol 2006;46(3):167-192. DOI: 10.1016/ j.yrtph.2006.06.002.

7. Sedighinia F, Safipour A, Ghazvini K. Antibacterial activity of Glacyrrhiza glabra against oral pathogens: an in vitro study. Avicenna J Phytomed 2012;2(3):118-124.

8. Messier C, Epifano F, Genovese S, et al. Licorice and its potential beneficial effects in common oro-dental diseases. Oral Dis 2012;18(1):32-39. DOI: 10.1111/j.1601-0825.2011.01842.x.

9. Korhalkar A, Deshpande M, Lele P, et al. Pharmacological studies of Yasthimadhu (Glycyrrhiza glabra) in various animal models-a review. Global J Res Med Plants Indigen Med 2013;2(3):152-164.

10. Prusti A, Misha SR, Sahoo S, et al. Antibacterial activity of some Indian medicinal plants. Ethnobotanical Leaf 2008;12:227-233.

11. CLSI. Performance standards for antimicrobial disk susceptibility test. Approved standard M02-A, Wayne, PA: CLSI. Clinical and Laboratory Standards Institute, 10th ed., vol. 29; 2009. p. 1.

12. Singh J, Kumar A, Budhiraja S, et al. Ethnomedicine: use in dental caries. Braz J Oral Sci 2007;6:1308-1312.

13. Korhalkar A, Deshpande M, Lele P, et al. Antimicrobial activity Of Yasthimadhu (Glycyrrhiza glabra L.): a review. Int J Curr Microbiol Appl Sci 2014;3(1):329-336.

14. Geetha RV, Roy A. In vitro evaluation of antimycotic activity of ethanolic extract of Glycyrrhiza glabra. Asian J Pharm Clin Res 2013;6(3):205-207.

15. Asl MN, Hosseinzadeh $\mathrm{H}$. Review of pharmacological effects of Glycyrrhiza sp and its bio active compounds. Phytother Res 2008;22(6):709-724. DOI: 10.1002/ptr.2362.

16. Pallavi SK. Effect of chlorhexidine on mutans streptococci and dental caries. J Indian Assoc Public Health Dent 2011;17:678-683.

17. Jenkins GN. Review of fluoride research since 1959. Arch Oral Biol 1999;44(12):985-992. DOI: 10.1016/S0003-9969(99)00110-7.

18. Hiremath SS. Textbook of public health dentistry, 3rd ed. New Delhi: Elsevier; 2016. ch. 36: Flourides. pp. 314-315.

19. Megalaa N, Thirumurugan K, Kayalvizhi G, et al. A comparative evaluation of the anticaries efficacy of herbal extracts (Tulsi and Black myrobalans) and sodium fluoride as mouthrinses in children: a randomized controlled trial. Indian J Dent Res 2018;29(6):760-767. DOI: 10.4103/ijdr.IJDR_790_16.

20. Asl Aminabadi N, Balaei E, Pouralibaba F. The effect of $0.2 \%$ sodium fluoride mouthwash in prevention of dental caries according to the DMFT index. J Dent Res Dent Clin Dent Prospects 2007;1(2):71-76. DOI: $10.5681 /$ joddd.2007.012.

21. Kulkarni VV, Damle SG. Comparative evaluation of efficacy of sodium fluoride, chlorhexidine and triclosan mouth rinses in reducing the mutans streptococci count in saliva: an in vivo study. J Indian Soc Pedod Prev Dent 2003;21(3):98-104.

22. Neko-Uwagawa Y, Yoshihara A, Miyazaki H.Long-term caries preventive effects of a school-based fluoride mouth rinse program in adulthood. Open Dent J 2011;5:24-28. DOI: 10.2174/1874210601105010024.

23. Ahn SJ, Park SN, Lee YJ, et al. In vitro antimicrobial activities of 1-methoxyficifolinol, licorisoflavan A, and 6,8-diprenylgenistein 
against Streptococcus mutans. Caries Res 2015;49(1):78-89. DOI: 10.1159/000362676.

24. Segal R, Pisanty S, Wormser R, et al. Anticariogenic activity of licorice and glycyrrhizine $\mathrm{I}$ : inhibition of in vitro plaque formation by Streptococcus mutans. J Pharm Sci 1985;74(1):79-81. DOI: 10.1002/ jps.2600740121.
25. $\mathrm{Hu} \mathrm{CH}, \mathrm{He}$ J, Eckert R, et al. Development and evaluation of a safe and effective sugar-free herbal lollipop that kills cavity-causing bacteria. Int J Oral Sci 2011;3(1):13-20. DOI: 10.4248/IJOS11005.

26. Jain E, Pandey RK, Khanna R. Licorice root extracts as potent cariostatic agents in pediatric practice. J Indian Soc Pedod Prev Dent 2013;31(3):146-152. DOI: 10.4103/0970-4388.117964. 